\title{
Towards Egocentric Way-Finding Appliances Supporting Navigation in Unfamiliar Terrain
}

\author{
Christopher Lueg and Nicola Bidwell \\ Charles Darwin University, Darwin, NT, Australia
}

christopher.lueg@cdu.edu.au nicola.bidwell@cdu.edu.au

\begin{abstract}
In this paper, we discuss foundations, requirements and first experiences with a mobile information system that supports, and is ecologically compatible with, human vision-based navigation and acquirement of spatial knowledge during movement through the physical world. The appliance assists a person finding his/her way from an origin to a destination by providing an egocentric (viewer-centered) rather than an abstract top-down map-type view of the surrounding environment. We illustrate the use of the application in a foreign, or partially familiar, built environment of the scale of a small town or university campus and discuss first field experiments exploring egocentric way-finding support.
\end{abstract}

Keywords: egocentric viewpoint, navigation, mobile information systems, PDA, appliance

\section{Introduction}

A person in a foreign environment wishing to walk to a place is often assisted by information communicated by persons more familiar with that environment that enables navigation to the destination by piloting. Piloting refers to performing a goal-directed path using landmark information in conjunction with an itinerary deduced from the spatial relations between current and destination locations (in: Golledge, 1999). Here, we discuss themes arising from developing a mobile information appliance that supports, and is ecologically compatible with, human vision-based navigation by piloting and acquirement of spatial knowledge whilst walking through the physical world. The appliance assists a person finding his/her way from an origin to a destination by providing an egocentric (viewer-centered), rather than an abstract top-down map-type, view of the surrounding environment. Supporting the user's natural predisposition for recognizing places and following paths between places helps circumvent some of the complexity issues associated with developing traditional virtual reality/mixed reality devices typically based on detailed threedimensional spatial models of the environment. Further, it may afford the potential for a community authored resource which includes the details of paths between destinations, and with reference to landmarks, provided by community members.

The paper is structured as follows. We first provide an illustrative scenario motivating this area of research. Then, we outline the theory behind the way-finding perspective we are exploring and discuss some design decisions influencing the prototype we have built. We conclude by discuss-

Material published as part of this journal, either on-line or in print, is copyrighted by Informing Science. Permission to make digital or paper copy of part or all of these works for personal or classroom use is granted without fee provided that the copies are not made or distributed for profit or commercial advantage AND that copies 1) bear this notice in full and 2) give the full citation on the first page. It is permissible to abstract these works so long as credit is given. To copy in all other cases or to republish or to post on a server or to redistribute to lists requires specific permission from the publisher at Publisher@InformingScience.org ing themes that have emerged from first field experiments exploring egocentric way-finding support and describing our research approach to address these. 


\section{A Usage Scenario - International Student Navigates Unfamiliar University Campus}

Compare, for formative purposes, two scenarios. In the first scenario, Lin, a new international student, arrives at the campus of an Australian university to attend her first lecture. Prior to beginning her journey she downloaded a campus map from the university's web site and printed the map on her ink jet printer. She hops off the bus at the university stop and now stands on the campus, facing an unfamiliar environment and a climate she may not be used to. Eager to get to the site of her first lecture, she starts navigating the university campus by comparing her continuous egocentric impression of her environment (which includes impressions of buildings, built structures, trees, clouds, and the blue sky) to an extrinsic abstract top-down view of the campus consisting of lines, rectangles and building numbers. Although Lin finally arrives at her chosen destination she has found it irritating to have had to abstract from her current location and constantly align two completely different perspectives, when comparing her impression of the campus environment to the abstract view map provided by the map.

An alternative personal digital assistant (PDA) - supported scenario might avoid forcing the user to constantly align two completely different perspectives in comparing the egocentric -naturalimpression of the physical environment to the abstract view map. In this scenario Lin, again a arrives at the campus of an Australian university to attend her first lecture as a new international student. Being familiar with using personal digital assistants (PDA) for various activities, she chooses, for navigation purposes, a digital way-finder application she has downloaded from the university's web site prior to beginning her journey. The way-finder contains geographic information of the university campus and its environs and images of distinctive features. The way-finder application appealed to Lin's sense of fun more than the traditional top-view map or mud-map on many university web-sites as its egocentric perspective is closer to the ego-shooters she loves to play than to those. Equipped with the PDA she hops off the bus at the university stop. Lin selects "university bus stop" when prompted by the way-finder to enter her current location, consults her electronic time-table (also downloaded from the university web site) and enters " 24 " and " 1 " as the building and room numbers of her destination lecture theater. The way-finder instructs Lin to look to her left and presents an image of two large white buildings where the corner of one obscures the other and there are a few steps leading up to a path in between. Lin looks across to her left to see the buildings which match those in the image and presses "OK". The way-finder returns the instruction for Lin to ascend the steps and follow the path between these buildings. At the same time it presents an image containing, in the upper half, a mirrored overhead building link bridge in the foreground and, in the lower half, a white sun canopy further away. As Lin rounds to the left of the nearer white building she notices the link bridge and some distance behind it a sun canopy. She proceeds to walk towards the link bridge and, when under it, presses "OK". The way-finder zooms into the sun canopy to indicate to that she should proceed directly to it and on reaching the sun canopy Lin presses "OK" again. The way-finder then instructs her to turn right onto the footpath beneath the canopy and follow this path until she reaches a junction in it, and presents the image of the corner of a building with distinctive red pillars. Lin proceeds along the footpath and, as she approaches the junction, presses "OK" when she notices the building with the red pillars. The way-finder presents the instruction to turn left at the junction in the footpath and presents an image containing a building which is partially obscured by a tree and another building in the background, on which there is a satellite dish. Lin turns to her left, follows the footpath and detects the satellite dish as it enters her view. She checks the way-finder again, notices she can click "OK" for the tree and, when she does so, is instructed to look left for building 24 . When Lin looks left she sees a sign by a door in a rather inconspicuous gray building declaring lecture theaters 24.01-24.09. Lin proceeds through this door and follows the signs to the lecture theater in time for her first class. 


\section{Research Background}

Our research into mobile information systems supporting egocentric navigation whilst walking in an environment is motivated by research into human navigation behavior by piloting. Piloting refers to performing a goal-directed path using landmark information in conjunction with an itinerary deduced from the spatial relations between current and destination locations (in: Golledge, 1999). People first acquire the spatial knowledge to navigate successfully from their direct experience of an environment rather than symbolically (Schacter \& Nadel, 1991). This knowledge allows humans to navigate transparently, without reference to symbols, canonical directions, or alignment with the Euclidean properties of the world (e.g. distance walked). Humans walking in the world draw upon allocentric (object-centered) and egocentric (viewer-centered) frames of reference from perspectives that are independent or relative to their vantage point, respectively. Unlike the extrinsic referents of maps these do not require orientation knowledge.

Two methods which, alone or combined, may sensibly provide mobile navigation at the scale of interest are based on landmarks and/or route knowledge. In navigation by landmarks salient distinctive visual cues at intermittent points along a route of travel are used to determine both the path to the destination and current location relative to the path (Wickens, 1992). The importance of humans' conception of space as a collection of familiar landmarks has been emphasized behaviorally, for example for newcomers to a city (Lynch, 1960; Thorndyke, 1980), and cognitively (Siegel \& White, 1975; Maguire, Burgess, \& O'Keefe, 1999). Route knowledge requires spatiotemporally relating a specific sequence of landmarks (e.g. the steps taken to move between landmarks) into a chronotopic structure. People learn the order of landmarks in a route very rapidly and earlier than the landmark's actual location.

Narratives of spatial layout suggest how frames of reference vary with the scale of a person's experience in the environment. People tend to describe groups of rooms and buildings in towns allocentrically by mental route and features of a room egocentrically by "gaze tours" with deictic references, such as "in front of" or "to the left of" (Taylor \& Tversky, 1996). In contrast, people tend to describe larger features of towns by "survey" using canonical direction terms (e.g. North, South). It takes significant time for people to configure a cognitive map (Tolman, 1948) of their survey knowledge, not least because space on a larger scale requires longer exploration. This internal representation entails constructing a mental model of the interrelationships among places with extrinsic frame of reference. The mental model, which may be map-like (e.g. Pezdec, 1983) or conceptually more abstract (e.g. McNamara, 1991), is imprecise and qualitative and requires association with landmarks to act as an index (in: Golledge, 1999).

\section{Design and Development Issues}

The majority of mobile navigation systems supporting a person walking seem to focus on an extrinsic perspective and appear better suited to larger scale navigation or assisting a person with some familiarity with the location. For example, systems implemented as head-mounted displays, such as WalkMap (Suomela, Roimela, \& Lehikoinen, 2003) or handheld devices, such as Cyberguide (Abowd et al., 1997), pictorially represent the user's position on a map. Limited representations of an intrinsic perspective of the environment have been implemented in the mobile tour systems GUIDE (Cheverst, Davies, Mitchell, \& Friday, 2000) and Tourist Guide (Simcock, Hillenbrand, \& Thomas, 2003). In these, digital images of key tourist attractions were used to relate a user's perspective to the extrinsic perspective map.

We propose a way finder application which presents route information between images of landmarks, presented as they would be seen when walking. Relative and intrinsic, rather than extrinsic, perspectives of the environment appear better suited to the $50 \%$ of people preferring to 
memorize and use route information rather than maps (Ohmi, 1998) and are more "ready-to-hand when situated in the context of walking.

Relative or intrinsic representational perspectives may reduce the irritation, mentioned in scenario (1), and the cognitive steps required to abstract from a person's mental model based on their current perspective of the real world in order to align it with a traditional map's perspective. They may also assist in circumventing current limitations of global positioning systems (GPS) such as their reliability and accuracy in built-up (e.g. Flintham et al., 2003). Further, representing the environment from this perspective might allow using the appliance in areas that are not yet covered by sufficiently detailed maps (e.g., Kimberly, Kakadu, Arnhem Land as examples of remote areas in Australia) or where extrinsic maps provide only limited support due to geographical constraints. An example of the latter might be environmental information lying on a vertical plane, such as on the walls of canyons, or underground, such as in caves.

Combining images and route information may extend possibilities for sharing navigation information. Individuals in a community, such as university staff or students, could contribute navigation information by uploading to the web route information to their choice of destinations that relates to a local library of landmark images. Relative and intrinsic perspectives of the environment appear to be less prone to the inter-subjective and cultural constraints of a map's symbolic traditions. Further, customization of the resource offers adaptability to cultural and language biases for egocentric or allocentric referents (e.g. Levinson, 1996).

There are a variety of research and development issues to be addressed. Situated navigation using a sequence of landmark images has physical contextual adaptation challenges. The system must account for the dynamic nature of the allocentric frames of reference afforded to a person in the real world and the topographically relevant information which lies beyond, or is obscured from, one field of his/her view. The formative scenario illustrates examples of these.

Consider a simple case which assumes the system had accurate information on both a user's position, for example by his/her confirmation on reaching the footpath junction, and body orientation, for example from input from an integrated flux compass. Even without the user re-orienting his/her head they may look at any $25^{\circ}$ of the horizontal subtent of the environment's image. This represents only $25-50 \%$ of the panorama captured by wide-angle ( $20 \mathrm{~mm}$ focal length) or normal (50mm focal length) lenses. Thus, the system might need to prompt the user to direct their focus towards a specified region if they are to notice landmarks, lying at different positions, heights and distances. The sun canopy mentioned in the second scenario is an example of a distinct landmark which also provides an obvious route across campus, however its structure obscures other landmarks while a person walks beneath it. Two otherwise similar orientations, displaced only a few meters apart, provide either a clear view of the satellite dish landmark or one obscured by a tree.

\section{Research Issues Coming Up During Our Investigations}

Various issues, bearing upon design and development, and requiring further exploration have emerged while we prepared and researched the usage scenario outlined. These include:

\section{Physical Constraints in Capturing and Displaying Digital Images and Sensory Constraints in Viewing Digital Images on a PDA}

Landmarks in virtual environments need to be realistic if they are to be transferable to navigation in real places (see: Maguire et al., 1999). Photographic images record light intensity and wavelength non-veridically and compress contrast, while a PDA's rendering capability has limited resolution and field of view. Some of the visual cues humans use when viewing the world, such as the optical flow field, motion parallax, binocular disparity are absent from still images. 


\section{Changes Regarding Features of the World}

Time of day, weather conditions, presence of temporary and seasonal features, including people, flora and fauna, can significantly alter the real world image.

\section{Significance of Features in the World}

Functionally more significant, in addition to physically larger, environmental features are prioritized for learning landmarks (Taylor \& Tversky, 1996). It is possible this may relate to what a person notices. If this is the case different choices of landmarks may be more appropriate for different people and/or for their different information seeking tasks.

It is of interest to discover the extent to which landmarks used to guide a path to a destination may also be used to guide a return to the origin given the different features apparent when reoriented by $180^{\circ}$. For example, the return route from the destination discussed in the scenario yields quite different views of the world.

\section{Significance of Features in Natural and Built Environments}

A built environment such as a town or university campus presents an image with a distinctive structure featuring places connected by paths or roads and dominated by right angles. Distinctive landmarks in such environments are likely to have qualitative differences to those in natural landscapes. Further, the characteristics of landmarks in a university campus, which has a relatively cohesive and homogeneous architecture engineered to support pedestrians differ from those in towns which serve diverse purposes and have evolved by gradual synthesis. For example, the landmarks in older cities have been found to be more easily remembered than those that have a modern, suburban-type, planning (Lynch, 1960).

\section{Impact of Different Ways of Navigating and Moving within Physi- cal Environments}

Specific ways of moving around in physical environments may be influenced by environmental conditions, such as weather conditions (e.g., people may try to avoid direct exposure to the sun in countries like Australia) or other environmental conditions (e.g., avoiding groups of people blocking the footpath; avoiding puddles after rainfall).

\section{Complementarity between Knowledge Supporting Navigation that is In Situ and Mobile}

Characteristics of the route information communicated to a user should be compatible with the route information available in the environment. An autonomous and relatively unified environment, such as university campus, tends to use consistent naming and signposting conventions for its buildings. This contrasts with a town which contains a mixture of standard and idiosyncratic naming and signposting practices.

\section{Ways to Capture a Person's Visual Impressions that are Rele- vant to Way-Finding}

There are various ways to capture a person's impression of the environment that are relevant to landmark navigation and can be re-used by a way-finding application. We are currently taking a phenomenological approach in which a person who has recently attempted to use images of landmarks to navigate creates further images of the same environment to guide another person. Such an approach complements and can be used to contextualize the results of techniques such as tracking the focus of a person's gaze as they explore their environment use eye-trackers. 


\section{Current Work}

In previous sections, we motivated the need for egocentric appliances by providing an illustrative scenario and we discussed the theory behind our work. We also raised a number of important issues to be investigated. Our work proceeds in two inter-related directions:

\section{A Research Framework for Exploring Support for Egocentric Navigation of Outdoor Environments}

We are working on a framework supporting the structured investigation of the issues raised in the previous section.

(1) Physical constraints in capturing and displaying digital images and sensory constraints in viewing digital images on a PDA

(1)Changes regarding features of the world

(1) Significance of features in the world

(10) Significance of features in natural and built environments

(1) Impact of different ways of navigating and moving within physical environments

(1) Complementarity between knowledge supporting navigation that is in situ and mobile

(1) Ways to capture a person's impression of way-finding such that the knowledge and, in particu-

lar, relevant visual impressions can be re-used in a way-finder application

Also, the framework will allow a more structured comparison of our work, pursuing a specific perspective building on scholarly work in biology, and related work, such as Cyberguide (Abowd et al., 1997) and GUIDE (Cheverst et al., 2000), reported in the more technically-oriented ubiquitous computing literature

\section{Building and Testing Support for Egocentric Navigation of Out- door Environments}

The work on the research framework is driven and complemented by building and field-testing prototypes realizing support for allocentric navigation of outdoor environments. Data collected during development and field-testing is used to extend, refine and verify the research framework discussed above.

Implementation platforms are a HP Tablet PC TC1000 and, later on, a HP H5450 personal digital assistant (PDA). A first prototype running on the Tablet $\mathrm{PC}$ has been developed by the second author and has been field-tested using the first author as subject. It is important to note that at that time, the subject was neither involved in the development of the prototype nor familiar with the part of the campus environment chosen as testbed. The prototype uses MS Windows Explorer slide show viewer to present a series of images of landmarks each preceded by a textual route instruction. All digital photos were taken with a Kodak CX330 3.1 megapixels camera with 3x optical zoom. Setting for the photos used in the walkthrough was on "landscape".

The actual test was similar to the student scenario described earlier in this paper. We used a protocol which was as naturalistic as possible; a second one concentrating on fewer variables had been prepared and will be used in the near future. The author who developed the appliance also prepared an itinerary of landmarks and textual instructions for the origin-destination path. Images of landmarks are drawn from a set of 50 photographs depicting 30 landmarks around the university campus. The talking aloud protocol was used during the task and in post-task analysis. 
We are currently preparing further experiments involving six users, with differing familiarity with the university campus, undertaking two short trips supported by a set images and instructions. These people answer a series of brief questions, printed on the reverse of each image or instruction, and record any additional observations. At the end of the way-finding session the users discuss their experiences and observations in a facilitated workshop.

Last but not least we are working on setting up an outdoor game involving students from different faculties. A game of "pilots and orienteers" will be used as a situated protocol to initiate our research into the nature of images of landmarks as communication tools. The object of the game is for the orienteers to follow a pilot's intended route, by using a sequence of photographs taken by pilots across a specified distance anywhere in the university campus. Orienteers will answer a standard set of brief questions, printed on the reverse of each image and record any additional observations. At the end of the way-finding game pilots and orienteers will discuss their experiences and observations in a facilitated workshop.

A future extension might involve a "pilots and orienteers" web site allowing different parties to construct path descriptions using a set of images provided. The pictures will be shown to subjects in a pre-defined way to avoid that they go directly to the target destination using their local knowledge of the campus. Incentives will be provided to those parties constructing paths that were successfully navigated by other parties not involved in the construction.

In addition to being fun, the data collected during the outdoor game will allow the investigators to compare the specific characteristics of path descriptions created ex situ while sitting at a computer and path descriptions created in situ while actually navigating the path to be described.

\section{Conclusions}

In this paper, we discussed an egocentric (viewer-centered) way-finding appliance supporting navigation in the real physical world. Research into egocentric way-finding appliances is motivated by scholarly work regarding the biological foundations of human navigation behavior. In particular, we explained the research background of this particular and we provided a usage scenario illustrating the benefit of using such an appliance. We then discussed issues encountered in our current research and during the development and field-testing of prototypes.

What we have accomplished to far is providing motivation, scientific foundations and first results justifying the research outlined in this paper. We proceed by developing a framework supporting the structured investigation of the issues raised and by further developing and testing the prototypes under development, using the data collected to extend, refine and verify the research framework. The game of "pilots and orienteers" will allow us to better understand the nature of images of landmarks as communication tools and the specific differences between path descriptions created ex situ while sitting at a computer and path descriptions created in situ while actually navigating the path to be described.

\section{Acknowledgments}

The equipment used was given to Charles Darwin University as part of a HP Philanthropy grant.

\section{References}

Abowd, G.D., Atkeson, C.G., Hong, J., Long, S., Kooper, R., \& Pinkerton, M. (1997). Cyberguide: A mobile context-aware tour guide. Wireless Networks, 3 (5), 421-433.

Cheverst, K., Davies, N., Mitchell, K. \& Friday, A. (2000). Experiences of developing and deploying a context-aware tourist guide: The GUIDE project. Proceedings of MOBICOM 2000. ACM. 
Flintham, M., Anastasi, R., Benford, S., Hemmings, T., Crabtree A., Greenhalgh, C., Rodden T., Tandavantitj N., Adams M., \& Row-Farr J. (2003). Where on-line meets on-the-streets: Experiences with mobile mixed reality games. Proceedings of the Annual ACM SIGCHI Conference on Computer Human Interaction, pp. 569-576.

Golledge, R.G. (1999). Precis of "Wayfinding behavior: Cognitive mapping and other spatial processes." PSYCOLOQUY 10(036) ftp://ftp.princeton.edu/pub/harnad/Psycoloquy/1999.volume.10/ psyc.99.10.036.cognitive-mapping.1.golledge http://www.cogsci.soton.ac.uk/cgi/psyc/newpsy?10.036

Levinson, S. (1996). Frames of reference and Molyneux's question: Cross-linguistic evidence. In P. Bloom, M. Peterson, L. Nadel and M. Garrett (Eds.), Language and space, pp. 109-69. MIT Press.

Lynch, K. (1960). Image of the city. MIT Press.

Maguire, E.A., Burgess, N. \& O'Keefe, J. (1999). Human spatial navigation: Cognitive maps, sexual dimorphism and neural substrates. Current Opinion in Neurobiology, 9, 171-177.

McNamara, T.P. (1991). Memory view of space. Psychology of Learning and Motivation, 27, 147-186.

Ohmi, M. (1998). How are egocentric and exocentric information used to find a way in virtual environments? Proc. IIXth International Conference of Artificial Reality and Tele-Existence, pp. 196-201.

Pezdek, K. (1983). Memory for items and their spatial locations by young and elderly adults. Develop. Psych, 19 (6), 895-900.

Schacter, D.L. \& Nadel, L. (1991). Varieties of spatial memory: A problem for cognitive neuroscience. In R G Lister \& H. J. Weingartner, Perspectives on cognitive neuroscience. New York, Oxford.

Siegal, A.W. \& White, S.H. (1975). The development of spatial representations of large-scale environments. In H. Reese (Ed.), Advances in Child Development and Behaviour (Volume 10, pp. 10-55). New York: Academic Press.

Suomela, R., Roimela, K. \& Lehikoinen, J. (2003). The evolution of perspective in WalkMap. Personal and Ubiquitous Computing, 7 (5), 249-262.

Simcock, T., Hillenbrand, S.P. \& Thomas, B.H. (2003). Developing a location based tourist guide application. Workshop on wearable, invisible, context-aware, ambient, pervasive and ubiquitous computing Adelaide Australia. In C. Johnson, P. Montague, \& C. Steketee (Eds.), Conferences in Research \& Practice in Information Technology (Volume 21).

Taylor, H.A. \& Tversky, B. (1996). Perspectives in spatial descriptions. Journal of Memory and Language, $20(5), 483-496$.

Tolman, E.C. (1948). Cognitive maps in rats and man. Psychol. Rev. 55, 189-208.

Thorndyke, P.W. (1980). Performance models for spatial and locational cognition. Washington, DC: Rand.

Wickens, C.D. (1992). Spatial perception and cognition and the display of spatial information. In Engineering Psychology and Human Performance. New York Harper Collins.

\section{Biographies}

